\title{
Strong evidence that daily use of fluoride toothpaste prevents caries
}

\author{
How effective is fluoride toothpaste in preventing caries?
}

\begin{abstract}
Twetman S, Axelsson S, Dahlgren H, Holm AK, Kallestal C, Lagerlof $\mathbf{F}$, et al., Caries-preventive effect of fluoride toothpaste: a systematic review. Acta Odontol Scand 2003; 61:347-355
\end{abstract}

Data sources Medline, Cochrane Library, reference lists of identified articles and selected textbooks were used to source studies.

Study selection Selected studies were randomized or controlled clinical trials of at least 2 years' duration with caries increment in the permanent (DMFS/T) or primary $(\mathrm{dmfs} / \mathrm{t})$ dentition as the endpoint. Reports published in Danish, English, French, German, Italian, Norwegian, Spanish or Swedish were included. For multiply reported trials the one with the longest follow-up period was included.

Data extraction and synthesis Inclusion decisions and grading of the studies was performed independently by two of the authors. The main outcome was prevented fraction (PF). A pooled estimate was calculated for included studies.

Results Fifty-four studies were included. There was strong evidence for a caries-preventive effect of daily use of fluoride toothpaste compared with placebo in the young permanent dentition (PF, 24.9\%; standard deviation, $\pm 11.5 \%)$. Toothpastes containing $1500 \mathrm{ppm}$ of fluoride had a superior preventive effect (additional $\mathrm{PF}, 9.7 \%$; range 0-22\%) compared with standard dentifrices of $1000 \mathrm{ppm}$ fluoride. There was also strong evidence for higher caries reductions with supervised toothbrushing compared with unsupervised brushing. There was incomplete evidence regarding the effect of fluoride toothpaste in the primary dentition.

Conclusions This review reinforces the importance of daily toothbrushing with fluoridated toothpastes for preventing dental caries, although long-term studies in age groups other than children and adolescents are still lacking.

\section{Commentary}

This is an important paper which highlights the strength of evidence supporting the daily use of fluoride toothpaste. Data were drawn from studies conducted in different geographical locations as well as in various age groups of children and adolescents. A clear benefit was observed, in terms of the preventive effect of fluoride, in studies that compared fluoride-toothpaste test groups with intervention controls that used a placebo or other fluoride-free toothpastes, with or without supervised brushing, in the young permanent dentition. In general, studies that used supervised brushing showed a higher preventive effect than those with unsupervised interventions.

Brushing is an important measure in prevention of caries because it contributes to the disruption of the dental biofilm. Evidence is still lacking, however, to show the isolated effect of brushing in the prevention of dental caries. This is probably because studies would be considered unethical if they compared treatment with control groups that did not use any preventive method at all. Nevertheless, future research is needed to measure the real effect of brushing, supervised or unsupervised, as an isolated variable from the effect of fluoride.

It has been suggested that the use of dentifrices with low fluoride concentrations $(<1000 \mathrm{ppm})$ for children under 6 years of age may reduce fluoride ingestion and risk of dental fluorosis. The effectiveness of these is unclear, however, and not supported by strong clinical, scientific evidence. Perhaps there should be consideration, therefore, of the best way to promote the use of toothpastes containing higher fluoride concentrations, in appropriate quantities, since these have been demonstrated to be more effective in controlling or reducing the risk of fluorosis in children. This paper also highlights the need for further studies concerning the effect of fluoride toothpaste in the primary dentition as well as in adults.

\section{Practice point}

- Practitioners should emphasise the importance of supervised brushing.

\section{Gail Topping and Andrea Assaf \\ Dental Health Services Research Unit, University of Dundee, Dundee, Scotland, UK}

Evidence-Based Dentistry (2005) 6, 32.

doi:10.1038/sj.ebd. 6400320 